\title{
Hot gas morpoloy, thermal structure, and the AGN connection in elliptical galaxies
}

\author{
Thomas S. Statler ${ }^{1}$ and Steven Diehl ${ }^{1,2}$ \\ ${ }^{1}$ Astrophysical Institute, Ohio University, Athens, OH 45701, USA \\ email: statler@ohio.edu \\ ${ }^{2}$ Los Alamos National Laboratory, Los Alamos, NM 87545, USA
}

\begin{abstract}
Recent studies of the hot interstellar medium in normal elliptical galaxies have shown that (1) the gas is only approximately hydrostatic; (2) morphological disturbances are corrleated with radio and X-ray signatures of AGN; and (3) temperature gradients in the main bodies of the galaxies are correlated with nuclear activity but not with environment. An X-ray Gas Fundamental Plane (XGFP), unrelated to the stellar fundamental plane, links the global gas properties in a relation whose origin is not yet understood.
\end{abstract}

Keywords. cooling flows; galaxies: elliptical and lenticular, cD; galaxies: ISM-X-rays: galaxies

\section{Introduction}

Current questions regarding the X-ray gas in elliptical galaxies concern modes of heating, coupling to central AGN, and even whether the gas is hydrostatic. This last is nothing new; even in the Einstein era Trinchieri et al. (1986) noticed the asymmetry of NGC 4472, and argued that the gas is not hydrostatic at large radii.

The early years of Chandra saw several in-depth studies of single, interesting, usually luminous, objects that quickly became "poster children" for AGN-driven feedback. Jones et al. (2002) argued that features in NGC 4636 are shocks created by intermittently-fueled AGN. Finoguenov \& Jones (2001) observed a striking H-shaped structure in NGC 4374, correlated with, and likely created by, expanding radio lobes.

In one of the few early studies of an X-ray faint system, Sarazin et al. (2001) demonstrated that low-mass X-ray binaries (LMXBs) contribute much of the total emission from NGC 4697. After removing the resolved sources, a diffuse component remained, apparently flatter than the starlight; however these authors emphasized that the gas morphology was uncertain because of the unknown contribution from unresolved LMXBs.

\section{Hot ISM Properties of Normal Ellipticals}

Diehl \& Statler (2007) (hereafter DS1) demonstrate that the emission from gas alone can be isolated by taking a suitably chosen scaled difference of hard-band and soft-band images. DS1 produce gas-only images for 54 normal ellipticals observed by Chandra, and obtain isophotal ellipticity and position angle profiles for each system as well as an "asymmetry index" measuring the deviation from a smooth elliptical model.

The angular resolution of Chandra allows measurement of gas ellipticities inside the optical effective radius $\left(R_{e}\right)$, where elliptical galaxies are stellar-mass dominated, offering a chance to test the assumption of hydrostatic equilibrium. DS1 find (their Fig. 6) that, contrary to this assumption, there is no correlation between optical and X-ray ellipticities. After extensive modeling, they conclude that the gas may be hydrostatic enough for radial mass profiles to be extracted, but it is not, in general, sufficiently hydrostatic for the shape of the gravitating mass to be inferred-asattempted by, e.g., Buote \& Canizares (1994) and Buote et al. (2002). 
The origin of morphological disturbances in the gas is addressed by Diehl \& Statler (2008a). They find that the asymmetry index $\eta$ is strongly correlated with the NVSS $20 \mathrm{~cm}$ luminosity and the X-ray luminosity of the central point source - signatures of AGN-and with the gas temperature gradient beyond $2 R_{e}$-an indicator of environment (their Fig. 11). Since $\eta$ measures small-scale asymmetries as well as large-scale lopsidedness, DS2 conclude that gas morphology is influenced in comparable measure by ram pressure in cluster environments and by AGN.

Unlike in galaxy clusters, gas temperatures in normal ellipticals do not always increase outward. Fukazawa et al. (2006) argue that positive radial temperature gradients indicate a transition to a hotter intragroup or intracluster medium. Humphrey et al. (2006) suggest that the gradient distribution is actually bimodal. Diehl \& Statler (2008b) show that there is no evidence for bimodality, and that the gradients inside $2 R_{e}$ are not affected by environment (as measured by the number density of neighbor galaxies), whereas the gradients outside $2 R_{e}$ are (their Fig. 5). They suggest that the change from positive to negative gradients reflects a change in the role or mode of AGN heating.

\section{X-Ray Gas Fundamental Plane}

The global properties of the stellar distribution in elliptical galaxies follow the well known Fundamental Plane: a 2-dimensional locus in the space of effective radius, surface brightness, and velocity dispersion that is primarily a consequence of the virial theorem. Diehl \& Statler (2005) find that, if one takes the analogous properties for the X-ray gas alone-half-light radius, X-ray surface brightness, and temperature, elliptical galaxies again delineate a plane, albeit a totally different one. The X-Ray Gas Fundamental Plane (XGFP) is not a consequence of the virial theorem, but is as tight as the stellar Fundamental Plane. Its origin has yet to be explained.

\section{Acknowledgements}

Support for this work was provided by the National Aeronautics and Space Administration (NASA) through Chandra Awards G01-2094X and AR3-4011X, and by National Science Foundation grants AST0407152 and AST0708284.

\section{References}

Buote, D. A. \& Canizares, C. R. 1994, ApJ 427, 86

Buote, D. A., Jeltema, T. E., Canizares, C. R., \& Garmire, G. P. 2002, ApJ 577, 183

Diehl, S. \& Statler, T. S. 2005, ApJ 633, L21

Diehl, S. \& Statler, T. S. 2007, ApJ 668, 150

Diehl, S. \& Statler, T. S. 2008a, ApJ 680, 897

Diehl, S. \& Statler, T. S. 2008b, ApJ 687, 986

Finoguenov, A. \& Jones, C. 2001, ApJ 547, L107

Fukazawa, Y., Botoya-Nonesa, J. G., Pu, J., Ohto, A., \& Kawano, N. 2006, ApJ 636, 698

Humphrey, P. J., Buote, D. A., Gastaldello, F., Zappacosta, L., Bullock, J. S., Brighenti, F., \& Mathews, W. 2006, ApJ 646, 899

Jones, C., Forman, W., Vikhlinin, A., Markevitch, M., David, L., Warmflash, A., Murray S., \& Nulsen, P. E. J. 2002, ApJ 567, L115

Sarazin, C. L., Irwin, J. A., \& Bregman, J. N. 2001, ApJ 556, 533

Trinchieri, G., Fabbiano, G., \& Canizares, C. D. 1986, ApJ 310, 637 